\title{
GÉNESIS SOCIOHISTÓRICA DE LA EXPRESIÓN CORPORAL EN ESPAÑA
}

\author{
SOCIAL-HISTORICAL GENESIS OF BODILY EXPRESSION IN SPAIN
}

GÊNESE SÓCIO-HISTÓRICA DA EXPRESSÃO CORPORAL NA ESPANHA

Daniel Caballero-Julia*, María Jesús Cuellar-Moreno**, Marie-Carmen Garcia*

Palabras clave:

Educación Física.

Políticas públicas.

Sociología.

Etnología.
Resumen: Este artículo tiene como objetivo clarificar y ordenar el proceso de institucionalización de la Expresión Corporal en España ante la escasez y fragmentación de investigaciones sobre los aspectos históricos que incidieron en las principales situaciones político-educativas y autores que la han configurado. Para ello, en primer lugar se ha realizado un análisis de documentos científicos y legales para profundizar en nuestro objeto de estudio. En segundo lugar, se han utilizado las entrevistas informales realizadas durante una observación etnográfica no participante y 16 entrevistas semiestructuradas. Como conclusión, la Expresión Corporal en España se encuentra condicionada por la situación histórica y por su sistema educativo. Además, se identifican geográficamente la Expresión Corporal del Noreste y la Expresión Corporal del Centro-Suroeste. A su vez, los autores han sido clasificados en dos generaciones que contribuyeron al proceso de creación, institucionalización y consolidación de la disciplina.

Palavras chave: Educação Física. Política pública. Sociologia. Etnologia.

Keywords: Physical Education. Public policy. Sociology. Ethnology.
Resumo: Este artigo tem como objetivo esclarecer o processo de institucionalização da Expressão Corporal na Espanha perante a escassez e a fragmentação das investigações sobre aspectos históricos que incidam sobre as principais situações políticas educativas e autores que a configuraram. Para isso, em primeiro lugar realizou-se uma análise de documentos científicos e jurídicos que permitem aprofundar o nosso tema de estudo. Em segundo lugar, recorreu-se às entrevistas informais realizadas durante uma observação etnográfica não participante e 16 entrevistas semiestruturadas. Como conclusão, a Expressão Corporal na Espanha encontra- se condicionada pela situação histórica e pelo seu sistema educativo. Além disso, identificam-se geograficamente a Corporação do Noroeste e a Corporação do Centro-Sudoeste. Por seu turno, os autores foram classificados em duas gerações que contribuíram para o processo de criação, institucionalização e consolidação da disciplina.

Abstract: This article explains and organizes the process of institutionalization of Bodily Expression in Spain considering the absence and fragmentation of research on historical aspects that impacted on the main political and educational situations and the authors that shaped it. To that end, scientific and legal documents were examined in order to elaborate on the object of study. Secondly, the study used informal interviews conducted during non-participant ethnographic observation and 16 semi-structured interviews. It found that Bodily Expression in Spain has been influenced by the country's historical context and educational system. Furthermore, it identified a Bodily Expression of the NorthEast and a Body Expression of the Centre and South-West. its authors were classified into two generations that contributed to the process of creation, institutionalization and consolidation of the discipline.
*Université Paul Sabatier. Toulouse França.

E-mail: daniel@caballero-julia.com; marie-carmen.garcia@univ-tlse3.fr

**Universidad de La Laguna. Santa Cruz de Tenerife, Espanha. E-mail: mcuellar@ull.edu.es

Recebido em: 20-07-2018 Aprovado em: 12-06-2019 Publicado em: 06-09-2019

DOI: hittps://doi.org/10.22456/1982-8918.85010 (c) (i) () Licence 


\section{PLANTEAMIENTO DEL PROBLEMA}

Para seguir el curso histórico de la Expresión Corporal (EC) en España es necesario indagar en sus orígenes, estando relacionados con gran cantidad de actividades corporales que conformaron este modelo de actividad física (MONTÁVEZ, 2012). Sus precursores provienen del mundo del teatro, la danza o la música, inspirándose en el ámbito de lo artístico.

Las diversas teorías sobre EC pueden dividirse en dos. Por un lado, una representación analítico-técnica del movimiento corporal y la actividad física más relacionada con la perspectiva científica (medicina, biología, etc.). Por otro, un enfoque artístico-educativo del que nace la actual EC que se encuentra relacionado con movimientos y actividades directamente influenciados por el mundo artístico (danza, teatro, música, etc.).

Romero (2015) indica que esta disciplina es considerada, incluso por los propios profesionales del género, como un cajón de sastre en el cabe cualquier tipo de actividad psicomotriz o expresiva. Ello afecta tanto a las posibles definiciones de este contenido como a las orientaciones del mismo, denominándola con el término de Actividades Corporales Expresivas (ACE) por su carácter entre Educación Física (EF) y Artes Escénicas y Plásticas. Cuéllar-Moreno y Pestano (2013), en un análisis de los planes de estudio de las Facultades de Educación respecto a la asignatura EC, indican que la denominación más utilizada es la de Expresión Corporal (41\%), seguida de la de Expresión y Comunicación Corporal (33\%).

Aunque en la actualidad existen numerosos libros y textos que versan sobre EC, se aprecia una carencia de información socio-histórica sobre este tema. Al mismo tiempo, la información existente resulta incompleta y se encuentra dispersa entre distintos textos. Por ello, en este trabajo nos planteamos como objetivo clarificar y ordenar el proceso de institucionalización de la EC en España ante la escasez y fragmentación de investigaciones. Para ello, se ha realizado una búsqueda de las bibliografías existentes para proseguir con la observación participante y la realización de entrevistas a expertos en la materia.

\subsection{ANTECEDENTES DE LA EXPRESIÓN CORPORAL}

A finales del siglo XIX y primeras décadas del siglo XX comenzó una renovación de la visión del cuerpo y de la actividad física. Tres escuelas tuvieron una influencia significativa en la creación de la EC: la escuela Francesa o Movimiento Occidental; la Escuela de Alemania o Movimiento del centro y la Escuela Sueca o Movimiento del Norte (MONTÁVEZ, 2012; ORTIZ, 2002). FIGURA 1.

La aparición de este movimiento regeneracionista también influyó en la política española al introducir un discurso sociopolítico y cultural de gran importancia para el impulso del deporte y la actividad física (RIVERO, 2009). El interés por regenerar la educación española se establece con la Ley Moyano (ESPAÑA. MINISTERIOR DE FOMENTO, 1857). Esta Ley de Instrucción Pública de 1857, considerada la primera Ley General de Educación. Su objetivo fue la reorganización administrativa de la educación española, hasta entonces caótica e incluso contradictoria. A pesar de su importancia mantuvo una base conservadora (BLANCO; DÁVILA; SARRAPIO, 2007).

La EF realizada hasta el comienzo de la dictadura de Primo de Rivera (1923-1930) se caracterizó por su baja calidad y se basó en los preceptos de la Escuela Sueca de movimientos 
muy estáticos y repetitivos. Además, promovió la educación para la patria, la mejora de la salud y la raza con un fuerte carácter militar (VIDAL BARBIER; VIDAL ALMIÑANA, 2008).

Figura 1 - Movimientos actuales de expresión corporal.

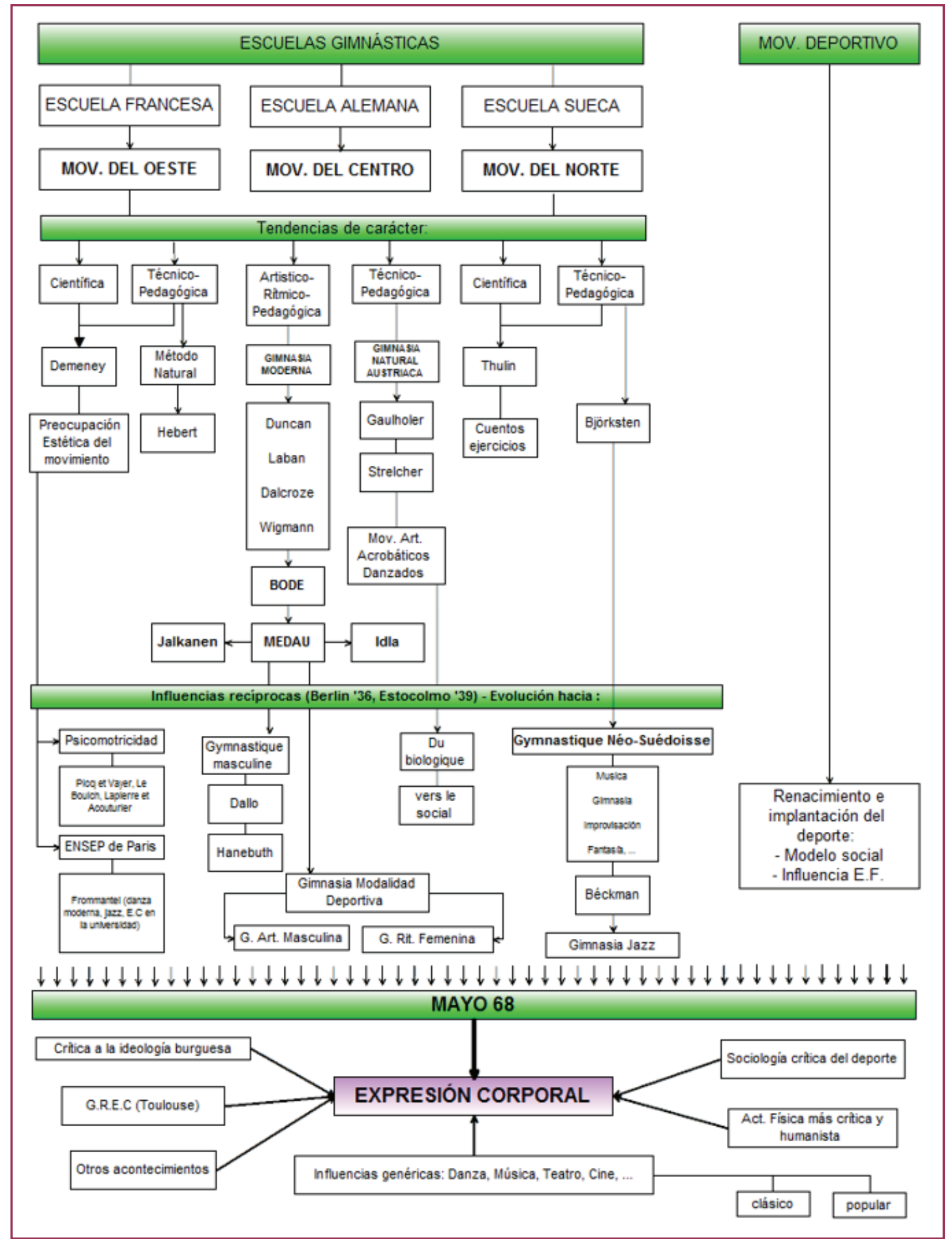

Fuente: ORTIZ (2002, p. 51)

La II República Española (1931-1939) modernizó el sistema político, productivo y social (CLARET, 2006), logrando insertar el deporte como el sustituto de la gimnasia sueca. La orientación de Ling hacia la salud coexistió en la educación republicana (VIDAL BARBIER; VIDAL ALMIÑANA, 2008). 
Las actividades corporales de expresión se caracterizaron en esta época por la separación entre el deporte y la gimnasia, coexistiendo en este ámbito una clara diversificación entre la gimnasia femenina y masculina. La femenina estaba muy influenciada por el mundo artístico orientado a incrementar la belleza y los roles de madres y esposas; por el contrario, la masculina tenía un enfoque higiénico y militar para vitalizar la masculinidad (GONZÁLEZ, 2009; MANRIQUE, 2003; MONTÁVEZ, 2012).

Durante la Guerra Civil Española (1936-1939) y la Dictadura Franquista (1939-1975), la política de represión tuvo prioridad en la educación estando ésta controlada por la Iglesia y el Movimiento (partido ideológico y político) que la transformó en un instrumento de control ideológico y de desmovilización de la sociedad. La Educación Primaria quedó separada por géneros, siendo obligatorio utilizar el castellano (DELGADO, 1994).

La EF se convirtió en un arma política como elemento de afirmación del régimen. Para los hombres tomó forma de curso basado en los preceptos de la Escuela Sueca (VIDAL BARBIER; VIDAL ALMIÑANA, 2008). Para la Sección de Mujeres se utilizaron los manuales para niñas diseñados por Luis Agosti, médico y deportista, siguiendo las instrucciones de la Escuela Neosueca (VIDAL BARBIER; VIDAL ALMIÑANA, 2008). Esta nueva escuela modificó la concepción de las actividades físicas mediante movimientos más fluidos y dinámicos (MARTÍN, 2009).

El Instituto Nacional de Educación Física (INEF) de Madrid fue creado en 1961 por la Ley de EF (ESPAÑA. JEFATURA DEL ESTADO, 1961), integrándose al mismo tras la dictadura la Academia de Comandantes que hasta entonces había gestionado esta asignatura (VIDAL BARBIER; VIDAL ALMIÑANA, 2008). Con esta Ley, se estableció la EF como asignatura obligatoria en todos los niveles de Educación. Por primera vez en la historia española, algunos contenidos relacionados con EC, como Medios de Expresión y Expresión Dinámica, se incluyen en la formación de profesores de EF en las instalaciones del INEF de Madrid siendo incluidos en el plan de estudios entre 1967-1968 y 1976-1977. Esta formación fue compartida con la Academia Nacional José Antonio que transmitió los contenidos más centrados en la teoría y la práctica de la Gimnasia Educativa, olvidando la Expresión Dinámica. Afortunadamente, el INEF de Madrid asimiló este último contenido en su programa educativo (COTERÓN; GIL, 2012), siendo el responsable de su inclusión el profesor, artista y músico Luciano González Sarmiento que vinculó este campo con el de la música (MATEU, 2010).

Patricia Stokoe, estudiante del maestro y precursor de danza moderna Rudolf von Laban fue de vital importancia en la creación de la EC en España. El comienzo de la Segunda Guerra Mundial supuso una experiencia crucial para comprender el carácter y la personalidad de Patricia Stokoe, así como para el papel de la EC en el aprendizaje de valores y para aprender a vivir la vida intensamente en cada momento (KALMAR, 2005; SÁNCHEZ, 2009).

En este contexto revolucionario, inspirado en el enfoque de Laban, Patricia Stokoe generó una pedagogía de la EC defendiendo tres principios fundamentales: la danza libre entendida como búsqueda de identidad propia inspirada en Laban, la danza accesible a todos y la danza entendida como una actividad que debe incluirse en el currículo de las escuelas, tal y como se había hecho en Inglaterra en las escuelas primarias.

Con el objetivo de ampliar su influencia educativa hacia más público, especialmente a la población masculina, decidió cambiar el nombre de sus actividades de Danza libre a 
Expresión Corporal (KALMAR, 2003; STOKOE, 1993, p. 1). La EC se convirtió en una apuesta por una nueva forma de insubordinación, así como una nueva forma de defensa de la libertad del individuo. La danza de Stokoe no estaba enmarcada en una técnica precisa o cuadrada, sino concebida como la expresión básica del lenguaje del individuo (SÁNCHEZ, 2009).

Para Stokoe, la EC tenía un espacio lo suficientemente grande como para considerarla como una disciplina diversa que podría orientarse hacia la educación o la terapia, siendo su principal motivación la democratización de la danza como lenguaje inherente del ser humano. Su metodología pedagógica se orientó, por lo tanto, hacia la incitación a la identidad, autoconsciencia y creatividad del estudiante. Obviamente, esta línea de pensamiento no estaba en consonancia con las ideas de educación transmitidas por el régimen de Franco, debiéndose esperar a la muerte del dictador para poder trasladar a España la EC de Stokoe.

\section{MÉTODO}

\subsection{MUESTRA}

La muestra está compuesta por 22 profesionales españoles de EC ${ }^{1}$. Concretamente, 6 docentes expertos ( 3 hombres y 3 mujeres) con más de 20 años de experiencia y 16 profesionales (14 mujeres y 2 hombres) de todo el territorio español. Estos pertenecen a la orientación pedagógica (13) y a la orientación artística (3) de la EC. El testimonio de los expertos fue obtenido a partir de la observación etnográfica, mientras que en los segundos se aplicó una entrevista semiestructurada.

\subsection{INSTRUMENTOS}

En este trabajo de investigación se han utilizado tres instrumentos. En primer lugar, se ha realizado una revisión bibliográfica de documentos científicos y legales para profundizar en nuestro objeto de estudio de manera histórica y política. En segundo, se analizaron las intervenciones a través de las entrevistas informales realizadas a 6 expertos en EC en el contexto de una observación etnográfica no participante. Estas intervenciones ayudaron a entender el origen y el significado de las prácticas observadas (ARBORIO; FOURNIER, 2015; LAPLANTINE, 2015). Por último, se realizaron 16 entrevistas semiestructuradas (BLANCHET; GOTMAN, 2007) a expertos en esta materia incidiendo sobre el papel de la EC en sus trayectorias profesionales.

\subsection{PROCEDIMIENTO}

Así, a partir de los datos recogidos, hemos extraído la información de cada uno de los instrumentos utilizados mediante un análisis puramente cualitativo. De este modo, hemos podido reconstruir el esquema social e histórico del proceso de institucionalización de la EC vivido en España. 


\section{RESULTADOS Y DISCUSSIÓN}

La idoneidad de nuestra muestra queda reflejada en la calidad de la información obtenida. Los textos indican datos específicos y objetivos sobre acontecimientos y normativas del pasado. La participación de los expertos nos ha permitido estructurar esta información de manera continua y coherente. Los profesionales entrevistados han puesto en relieve los papeles jugados por el conjunto de actores y su influencia sobre los profesionales de hoy en día.

\subsection{EL NACIMIENTO DE UNA NUEVA DISCIPLINA.}

En la España de 1970, como respuesta a la apertura del país que caracterizó a los años 60, se firmó una nueva Ley General de Educación (LGE) (ESPAÑA. MINISTERIO DE EDUCACIÓN Y CULTURA, 1970) en la que la EF continúo siguiendo el sistema Ling de la Escuela Sueca, con valoraciones morales y espirituales (VIDAL BARBIER; VIDAL ALMIÑANA, 2008). A pesar de ello, el desarrollo de la EC era ya un proceso imparable.

Según los expertos en EC Neus y Miguel la consideración de contenidos relacionados con la EC en la LGE representó un gran avance para esta disciplina, en particular por su inclusión en el plan de estudios del INEF de Madrid mediante la asignatura Expresión Dinámica. A este respecto, Coterón (2008) y Vázquez (1989) indican que la EC carecía de tradición educativa tanto en España como a nivel internacional (en Europa solo fue incluida como contenidos de danza o ritmo).

En esta misma línea, y coincidiendo con los expertos, Ríos (2006) indica que la EC adoptó la re-centralización del cuerpo como un medio de retorno a la naturaleza frente a los valores de grupos hegemónicos (HERNÁNDEZ, 1996), denominado corporeismo (MAISONNEUVE; BRUCHON, 1981). Éste incorporaba un ideal basado en la liberación sexual, comunicación espontánea y sentirse cómodo en su propia piel (RíOS, 2006, p. 68) que recoge la influencia extranjera de un contexto sociopolítico cultural muy agitado. De este modo, los movimientos culturales y sociales de las revueltas de mayo de 1968 reafirmaron el cuerpo actual de la EC como una manifestación social con contenidos y métodos propios, diferenciados, incluso con conexiones de la danza, la mímica o el teatro (ROMERO, 2015, p. 130).

Miguel, profesor experto de EC en una universidad pública, nos cuenta cómo en este contexto en España se inició una serie de relaciones interpersonales entre personas con una clara orientación artística, que aprovechando los cambios sociopolíticos crearon la EC que conocemos en la actualidad. Destaca entre ellas, Patricia Stokoe, como figura clave en la génesis de esta disciplina artística y física. Su danza era una danza para todos en oposición a los movimientos normados dirigidos únicamente a las mujeres.

\subsection{PRIMERA GENERACIÓN: LA CREACIÓN DE LA EXPRESIÓN CORPORAL ESPAÑOLA}

A finales de la década de los 60 entró en escena la argentina Ana Pelegrín, quien según nos cuenta Miguel (experto en activo y antiguo alumno suyo) fue miembro del cuerpo docente del INEF de Madrid donde desarrolló una red profesional de Actividades Corporales Expresivas junto con Stokoe, Schinca entre otros artistas, pedagogos y educadores (Figura 2). Su trabajo tuvo un gran impacto sobre los eventos formativos de los especialistas del territorio español. 
Figura 2 - Institucionalización de la Expresión Corporal en España (1970-1980).

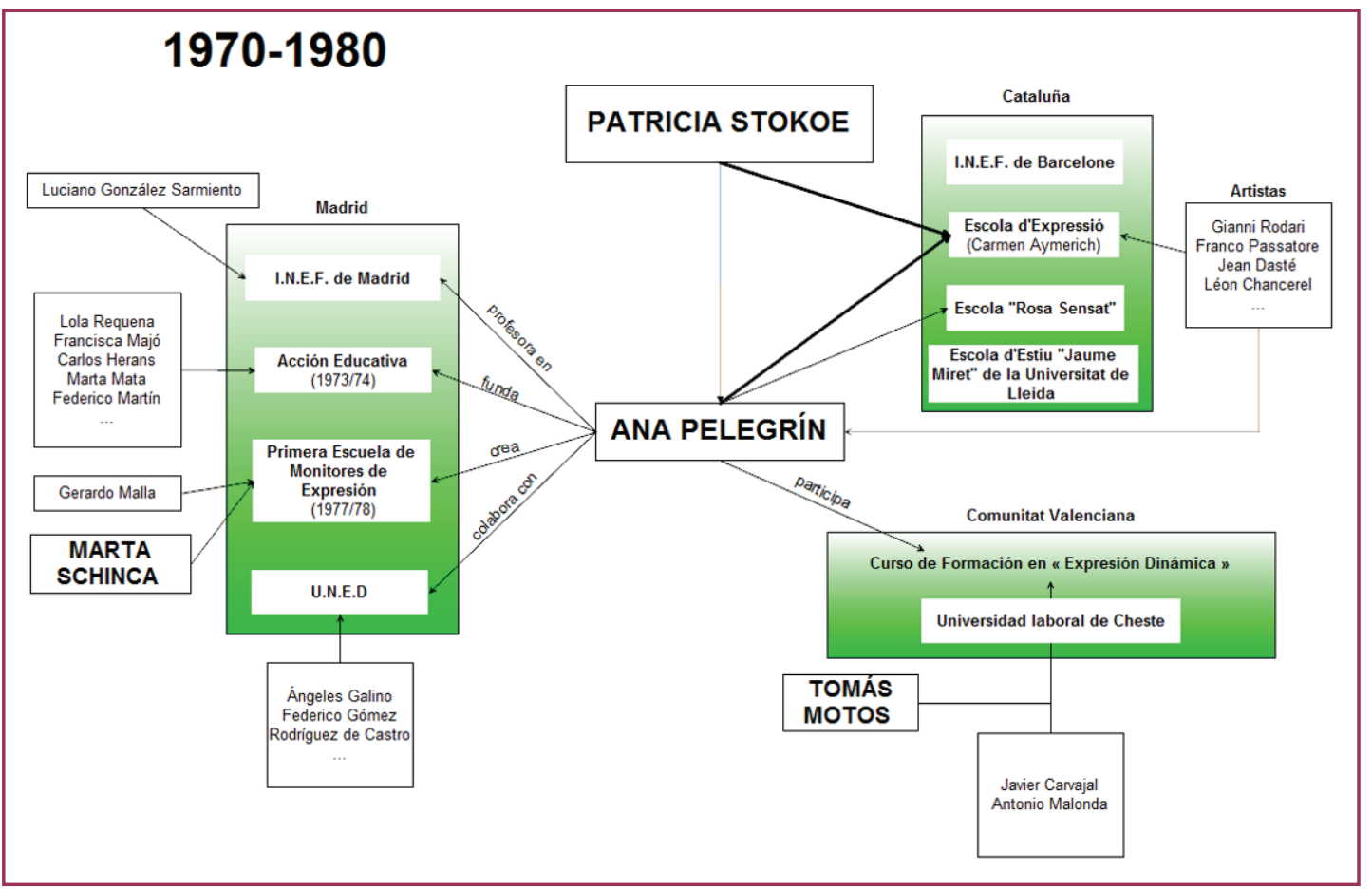

Fuente: elaboración propia a partir de los expertos y citas bibliográficas.

En colaboración con la Universidad Nacional de Educación a Distancia (UNED), creó numerosos materiales didácticos para el campo de los programas de estudio de Expresión Dinámica. Muy interesada en las formas de comunicación artística, estuvo involucrada en el primer curso de formación especializada en Expresión Dinámica que tuvo lugar en la Universidad Laboral de Cheste de Valencia. En el contexto de esta formación, Ana Pelegrín trabajó con profesionales como Javier Carvajal, Antonio Malonda y Tomás Motos, desarrollando este último especialmente los aspectos teóricos de la EC (SÁNCHEZ; COTERÓN, 2007).

Sánchez y Coterón $(2009 ; 2007)$ afirman que durante este período, Ana Pelegrín estableció una estrecha relación la Escuela Rosa Sensat y la Escuela Municipal de Expressió y Psicomotricidad de Barcelona. Ambas, situadas en Cataluña, representaron las primeras escuelas de formación en actividades artísticas y expresión de España. A través de estas colaboraciones, Pelegrín integra una visión multidisciplinar a sus contribuciones pedagógicas de la EC. El establecimiento de vínculos entre Pelegrín y la Escola de Expressió conectó las dos principales fuentes de actividad en torno a las Actividades Corporales de Expresión (ACE) donde nació la EC española (MATEU, 2010).

Los profesores Vidal y Vidal (2008) afirman que el inicio del período democrático trajo a la educación, y más concretamente a la EF, cambios de gran importancia a nivel administrativo y educativo. En primer lugar, y tras la muerte del dictador, se creó el segundo INEF de España en Barcelona. Este fenómeno tuvo como consecuencia la democratización de la disciplina y la separación del INEF de las estructuras educativas franquistas. La Ley General 13/1980 de Cultura Física y Deporte (ESPAÑA. JEFATURA DEL ESTADO, 1980) ubicó a la EF al mismo nivel que otras asignaturas educativas.

Sánchez y Coterón (2007) coinciden con Miguel al decir que durante este período de cambio y de creación de los INEF, Pelegrín desarrolló una red de contactos y reflexionó sobre 
los modos de mejorar la formación de sus estudiantes. Con objeto de ampliar la formación en la expresión artística y las actividades corporales, esta autora creó con Carlos Herans, la primera escuela de animadores de expresión. Esta capacitación tuvo una duración de dos años académicos y contó con la participación de Gerardo Malla y Marta Schinca, creando esta última su propia compañía de teatro Schinca Teatro de Movimiento en 1977, mediante la que transmitió el método Técnica y Expresión del movimiento.

\subsection{SEGUNDA GENERACIÓN Y CONSOLIDACIÓN DE LA DISCIPLINA}

El currículo educativo fue revisado por la Ley Orgánica de Ordenación General del Sistema Educativo (LOGSE) en 1990 (ESPAÑA. MINISTERIO DE EDUCACIÓN Y CULTURA, 1990), estableciendo tres niveles de concreción en su diseño: nivel nacional, regional y de centro escolar. Según Marchesi (2001), el enfoque pedagógico se orientó hacia la definición de objetivos educativos vinculando las habilidades de los estudiantes con un sistema de evaluación continua e inclusión de valores en la educación transversal.

Los INEF de Granada, Galicia, Canarias se crearon los primeros, tras el INEF de Barcelona, durante la década de los 80 . Ello permitió el incremento tanto a nivel cuantitativo (mayor número de profesores especializados en EF) como cualitativo (desarrollo de los contenidos formativos de los INEF) de la EF (LÓPEZ FERNÁNDEZ, 2003) y, con ello, de la EC.

Según Vidal y Vidal (2008), la LOGSE implicó una amplia extensión de la educación a toda la población española y en la revisión de la calidad educativa en el Sistema Educativo Español, contribuyendo al desarrollo institucional de los INEF y de la EF española. Esta Ley permitió, por primera vez, una integración completa de la EF en los Centros de Formación Docente, así como la posibilidad de incrementar el número de especialistas de EF en los diferentes niveles de educativos. Además, supuso la inclusión de la EF en Educación Primaria, que había sido abandonada hasta entonces por falta de recursos y personal.

Estos mismos autores nos recuerdan cómo, posteriormente, se incorporaron los INEF a la Universidad, consiguiendo con ello que la EF se integrara junto al resto de disciplinas que formaban parte del Ministerio de Educación y Ciencia. Por tanto, los estudios para entrenadores en actividad física y deporte, así como en actividades corporales de expresión se incluyeron en la Universidad. Esta inclusión, según los profesionales consultados, estuvo acompañada por la integración del contenido de EC no solo en los estudios de capacitación de los especialistas de Educación Física, sino en todos los niveles del Sistema Educativo Español. Además, los expertos afirman que la intervención de Marta Schinca fue decisiva. A partir de la publicación de su libro Psicomotricidad, ritmo y Expresión Corporal, Schinca y un grupo de colaboradores participaron en la redacción del contenido de EC de la LOGSE.

Ruano (2004) coincide que, como resultado de ello, los contenidos de Expresión Corporal se incluyeron explícitamente en EF en los niveles de Educación Primaria y Educación Secundaria.

Son numerosos los autores que han justificado la incorporación de estos contenidos en el currículo LOGSE por las funciones estéticas y comunicativas que se atribuyen a las actividades corporales artísticas y expresivas (REBOLLO; CASTILLO, 2010; ROMERO, 2015; RUANO, 2004). Al mismo tiempo, su presencia en el plan de estudios también ha sido justificada 
por el deseo de cambiar los modelos de EF (visión higienista, orientación al esfuerzo y el sector masculino, etc.) expresados en la LOGSE.

En este contexto, y anticipando la necesidad de una formación especializada en EC, Ana Pelegrín reunió a un grupo de exalumnos para establecer un espacio de reflexión y formación de especialistas de actividades físicas que mostraron un interés real en esta nueva disciplina. Como resultado en 1995, Pelegrín inició la organización de un Seminario Permanente en el INEF de Madrid. Este grupo no buscaba solo sus enseñanzas, sino todo tipo de entrenamiento que les permitiese acercarse a la EC. La mayoría de estos especialistas, viajaron por la geografía española y extranjera para aprender y recopilar todo tipo de información y capacitación sobre el movimiento corporal.

Estos seminarios permanentes persiguieron recoger y transformar la pedagogía del lenguaje corporal, promover actividades de investigación que permitieran la publicación de materiales de enseñanza relacionados con la disciplina y, en definitiva, promover la capacitación del personal docente en EC de todos los niveles educativos a nivel nacional (SÁNCHEZ; COTERÓN, 2007). Como producto, surge la asociación de Actividad Física y Expresión Corporal (AFYEC) que fue fundada en 1997, dos años después del inicio de los Seminarios Permanentes del INEF de Madrid. A pesar de la importancia del papel de Ana Pelegrín en la creación de AFYEC, la autora mantuvo un distanciamiento para dar un mayor protagonismo a sus discípulos, aunque participó del consejo de administración durante los primeros años hasta la consolidación del grupo y Asociación en 2002.

Figura 3 - Proceso de institucionalización de la expresión corporal en españa (1990-2010)

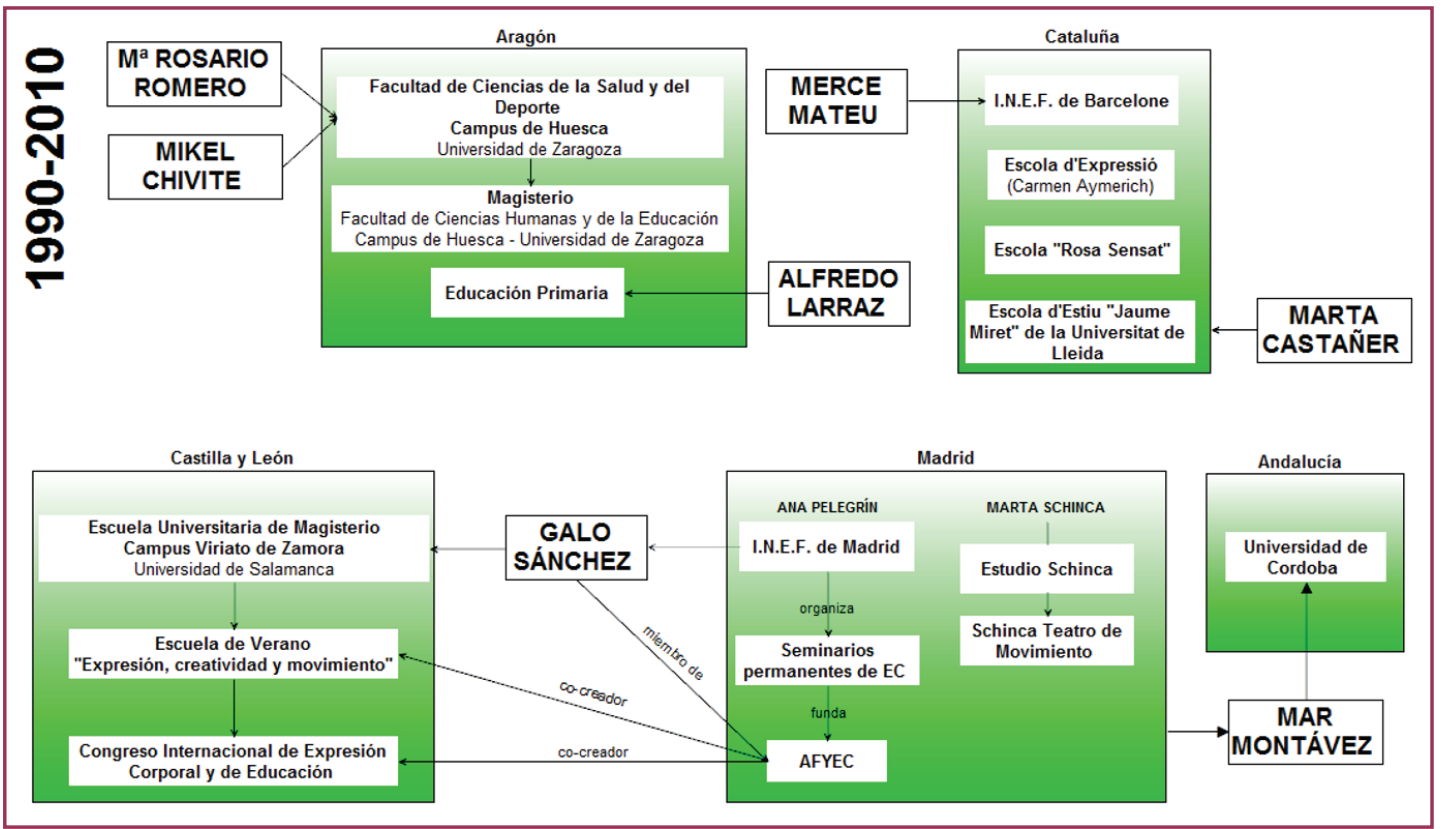

Fuente: elaboración propia a partir de los expertos y citas bibliográficas.

En la actualidad, AFYEC representa uno de los focos más importantes de la EC española que contribuye a la difusión y consolidación de la EC en el Sistema Educativo Español. Figura 3.

Según puede apreciarse en ciertas entrevistas como la de Susana o Belén, Galo Sánchez -profesor de la Escuela Universitaria de Magisterio de la Universidad de Salamancafue influido por el espíritu creativo de Ana Pelegrín (SÁNCHEZ, 2008). Inició así un fuerte trabajo 
de impulso de la EC, con el apoyo y la colaboración de AFYEC. En 1999, Sánchez organizó en Zamora el I Escuela de Verano, Expresión, Creatividad y Movimiento en las instalaciones del Campus de Viriato. Esta primera edición contó con el apoyo de Ana Pelegrín que fue incluida en el equipo docente de la Escuela, configurándose como un nuevo capítulo en la breve historia de la EC en España. Las diversas ediciones de la Escuela de Verano de Zamora han tenido un número creciente de profesionales y personas atraídas por las Actividades Corporales Expresivas, participando en ella numerosos y prestigiosos docentes de la EC como Alfredo Larraz, Kiki Ruano, Mar Montávez, Ana Pelegrín, Carmen Padilla, Leslie Kalmar (hija Patricia Stokoe), Mํㅡㄹ Rosario Romero, etc.

Seguidamente se organizaron con la colaboración de AFYEC dos Congresos Internacionales de EC y Educación en 2003 y 2008 (SÁNCHEZ, 2008), donde participaron Deborah y Leslie Kalmar (hijas de Patricia Stokoe), Ana Pelegrín, Marta Schinca, Merce Mateu y Tomás Motos, Alfredo Larraz, Tizou Pérez, Mํㅡㄹ Rosario Romero y muchas otras personalidades que representaron y representan a toda una generación de profesionales que han contribuido a la implementación de la EC en España. Muchos de estos participantes forman parte de lo que llamaremos aquí Expresión Corporal del Nordeste, estando entre las principales instituciones: el INEFC de Barcelona, el INEFC de Lleida y la Universidad de Zaragoza.

Desde el inicio del INEFC de Barcelona, en 1977, la Expresión Dinámica tomó una orientación más escénica dada la experiencia y el origen del profesor de la asignatura Pawel Rouba. En 1997, ya inmerso en el contexto legislativo de la LOGSE, los contenidos de Expresión Dinámica del INEF de Cataluña se integraron en el programa de Juegos bajo el nuevo nombre Teoria i Practica del Joc i l'Expressió Corporal. Rápidamente en las diferentes facultades universitarias los contenidos tomaron la forma de asignatura independiente bajo los nombres de Expresión Corporal, Expresión y Creatividad en movimiento, Movimiento y Expresión, entre otros. Fue un período de implementación de nuevas leyes pero también de nuevos contenidos. La formación de los futuros profesores tuvo que ajustarse a las características de los programas que limitaban el número de horas. La EC en este nivel estaba ganando terreno con la implementación de las asignaturas obligatorias en las facultades de Ciencias de la Actividad Física y el Deporte, así como en los INEF. La oferta se ha ampliado con asignaturas optativas, un gran número de cursos extraordinarios universitarios y cursos no adscritos a la Universidad (MATEU, 2010).

Según nos cuenta en sus trabajos y corroboran nuestros expertos, la profesora Mercè Mateu del INEFC Barcelona, abarca en su trabajo tanto aspectos teóricos como prácticos de la EC. A partir de 1992, Mateu organizó seminarios monográficos y cursos de formación sobre circo y Educación Física, que dieron lugar a publicaciones y representaciones teatrales que combinan los aspectos artísticos y educativos de los recursos del circo. En su tesis doctoral, Mateu (2010) destaca la creación, en 1988, del grupo Flip-Flop que trabaja con actividades que combinan técnicas expresivas con técnicas gimnásticas.

Paralelamente, podemos encontrar en la Universidad de Lleida el Taller de Danza para los Servicios Culturales a cargo de la profesora Marta Castañer, así como la creación del grupo Esbós Dansa-UDL desde 1985 y la organización de actividades con los ciudadanos de Lleida en 1997 con Mostra de Dansa (MATEU, 2010). Ma Rosario Romero y Mikel Chivite en el Campus Universitario de Huesca perteneciente a la Universidad de Zaragoza, contribuyen a la producción teórica del corpus de EC española produciendo numerosas obras para la disciplina. 
Y finalmente, pero no menos importante, expertos como Belén y Roke destacan el trabajo pedagógico y práctico de Alfredo Larraz (maestro de Educación Primaria en Jaca y docente de la Facultad de Ciencias Humanas y Educación en Huesca). Larraz jugó el papel de intermediario entre el lenguaje corporal francés y el español. Para él, la EC en España se desarrolló tardíamente en comparación con el progreso experimentado en Francia. Independientemente de los cambios políticos e incluso artísticos que siguieron en España desde la década de 1960, no tuvo dudas en ir más allá de las fronteras pirenaicas buscando algo nuevo, fresco en el estudio del cuerpo y la práctica expresiva. Por ello, dedicó su tiempo y recursos a conocer a artistas y autores que trabajaban estos contenidos en Francia conociendo así a Jean Le Boulch o Pierre Parlebas.

En Andalucía, según varios profesionales entrevistados, la profesora Mar Montávez, destaca con la realización de más de cien cursos de formación continua en el ámbito de la EC, organizados por el Instituto Andaluz del deporte, Centros de Profesores, Escuelas de Danza, Diputaciones y diversas universidades. Su obra incluye tres libros de carácter principalmente práctico, numerosos artículos y varios capítulos de libro.

\section{CONCLUSIONES}

En este trabajo nos planteamos como objetivo clarificar y ordenar el proceso de institucionalización de la EC en España. Para ello, se han analizado sus orígenes ante la escasez y fragmentación de investigaciones sobre aspectos históricos que incidan en las principales situaciones político-educativas y los autores que la han configurado.

Con el fin de profundizar en nuestro objeto de estudio, se ha realizado en un primer momento un análisis de los documentos científicos y legales existentes. Este análisis nos ha permitido establecer que la evolución de la disciplina aparece aparejada a la historia española y de su sistema educativo, configurando el desarrollo de la Educación Física.

En un segundo lugar, se ha realizado un análisis etnográfico y entrevistas a expertos en la materia. Ello nos permite dilucidar las diversas tendencias que existen sobre EC, constatándose la existencia de dos zonas geográficas diferenciadas y autores que contribuyen a su institucionalización. La primera de estas zonas ha sido denominada como la Expresión Corporal del Noreste y la segunda Expresión Corporal del Centro-Suroeste, cuyos focos históricos principales son el INEF de Barcelona y el INEF de Madrid, respectivamente.

En cuanto a los autores principales que la han conformado, pueden clasificarse en dos generaciones. La primera de ellas destacó por el nacimiento e institucionalización de la disciplina con autores como Patricia Stokoe, Ana Peregrín, Marta Schinca, Mercè Mateu y Tomás Motos. La segunda generación destaca por el proceso de consolidación de la EC con autores como Galo Sánchez, María del Rosario Romero, Mikel Chivite, Mar Montávez y todo el profesorado asociado a AFYEC.

Las aportaciones recogidas en el primero de estos análisis nos permiten analizar la evolución de la EC desde la dictadura de Primo de Rivera, pasando por la II República, Escuelas de gimnasia, Guerra Civil Española y dictadura Franquista. Se prosigue con la Ley General de Educación en 1970 y la creación y consolidación de la EC. Se busca pues, ofrecer nuevas perspectivas al estudio de un periodo y problemática que condiciona la continuidad de la EC en España. 
A pesar de nuestro esfuerzo por categorizar las diferentes zonas y generaciones, somos conscientes de la importancia de destacar la interrelación existente entre estos espacios geográficos, así como entre las generaciones de profesionales que hicieron posible el proceso de creación, institucionalización y consolidación de la EC en España.

Esperamos que con este estudio hayamos contribuido a una mayor comprensión de la EC en España que motive el desarrollo de nuevas investigaciones sobre la materia.

\section{REFERENCIAS}

ARBORIO, Anne-Marie; FOURNIER, Pierre. L'observation directe. 4. ed. Paris: Armand Colin, 2015. BLANCHET, Alain; GOTMAN, Anne. L'entretien. 2. ed. Malakoff: Armand Colin, 2007.

BLANCO, Ma Henar; DÁVILA, Susana; SARRAPIO, P. Evolución Histórica del Sistema Educativo Español. Educación Y Futuro Digital, v. 12, p. 1-2, 2007.

CLARET, Jaume. Cuando las cátedras eran trincheras. La depuración política e ideológica de la Universidad española durante el primer franquismo. HISPANIA NOVA Revista de Historia Contemporánea, n. 6, 2006. Disponível em: http://hispanianova.rediris.es/6/dossier/6d018.pdf. Acceso en: 29 sept. 2015.

COTERÓN, Javier. La Expresión Corporal en la Ley General de Educación de 1970. El Nacimiento de una Nueva Disciplina. In: SÁNCHEZ, Galo et al. (Eds.). Expresión Corporal, Investigación y Acción Pedagógica. Salamanca: Amarú, 2008. p. 165-173.

COTERÓN, Javier; GIL, Javier. La Expresión Corporal en los estudios en Ciencias de la Actividad Física y del Deporte. In: SÁNCHEZ, G.; COTERÓN, J. (Eds.) La Expresión Corporal en la Enseñanza Universitaria. Salamanca: Ediciones Universidad de Salamanca, 2012. p. 43-58.

CUELLAR-MORENO, María Jesús; PESTANO-PÉREZ, M. Avelino. Formación del Profesorado en Expresión Corporal : planes de estudio y Educación Física Training teacher in Body Expression : study program and Physical Education. Retos. Nuevas tendencias en Educación Física, Deporte y Recreación, p. 123-128, 2013. Disponível em: http://ucsj.redalyc. org/articulo.oa?id=345732290026. Acceso en: 29 sept. 2015.

DELGADO, Buenaventura. La educación en la España contemporánea (1789-1975). In: BUENAVENTURA, D. C. (coord). Historia de la Educación en España y América. Madrid: Morata, 1994. v. 3, p. 13-27.

ESPAÑA. JEFATURA DEL ESTADO. Ley 77/1961, de 23 de diciembre, sobre Educación Fisica. Boletín Oficial del Estado, Madrid: p. 18125-18129, 1961.

ESPAÑA. JEFATURA DEL ESTADO. Ley 13/1980, de 31 de marzo, General de la Cultura Física y del Deporte. Boletín Oficial del Estado, Madrid, p. 1-13, 1980.

ESPAÑA. MINISTERIO DE EDUCACIÓN Y CULTURA. Ley 14/1970, de 4 de agosto, General de Educación y Financiamiento de la Reforma Educativa. [Disposición derogada]. BOE» núm. 187, de 6 de agosto de 1970, páginas 12525 a 12546,Madrid, p. 22, 1970. Disponível em: https://www.boe.es/ boe/dias/1970/08/06/pdfs/A12525-12546.pdf. Acceso en: Acceso en: 29 sept. 2015. 
ESPAÑA. MINISTERIO DE EDUCACIÓN Y CULTURA. Ley Orgánica de Ordenación General del Sistema Educativo. (LOGSE). Boletín Oficial del Estado, Madrid, n. 238, 1990. Disponível em: http://www.jerez.es/fileadmin/Documentos/Biblioteca/Orden 30 Julio 2007.pdf. Acceso en: 29 sept.2015.

ESPAÑA. MINISTERIOR DE FOMENTO. Ley de Instrucción Pública (Ley Moyano). Gaceta de Madrid, Madrid, p. 1-3, 1857. Disponível em: https://www.boe.es/datos/pdfs/BOE/1857/1710/A0000100003.pdf. Acceso en: Acceso en: 29 sept. 2015.

GONZÁLEZ, Teresa. Los programas escolares y la transmisión de roles en el franquismo: la educación para la maternidad. Bordón, v. 3, n. 61, p. 93-105, 2009. Disponível em: https://dialnet. unirioja.es/descarga/articulo/3054923.pdf. Acceso en: Acceso en: 29 sept. 2015.

HERNÁNDEZ, Juan Luís. La Construcción Histórica y Social de la Educación Física: el currículo de la LOGSE, ¿una nueva definición de la educación física escolar? Revista de Educación, v. 311, n. 1, p. 51-76, 1996. Disponível em: http://www.mecd.gob.es/dctm/revista-de-educacion/articulosre311/ re3110300458.pdf?documentld=0901e72b81272f75. Acceso en: Acceso en: 29 sept. 2015.

KALMAR, Deborah. Con los ojos del corazón: Aportaciones principales de la pedagogía de Patricia Stokoe al desarrollo educativo de la Expresión Corporal. In: G. SÁNCHEZ, G. B.; TABERNERO, J.; COTERÓN, C; LLANOS ,Y.;. LEARRETA, B. (coord.) CONGRESO INTERNACIONAL DE EXPRESIÓN CORPORAL Y EDUCACIÓN: EXPRESIÓN, CREATIVIDAD Y MOVIMIENTO.1., 2003. Actas... Salamanca: Amarú, 2003. p. 31-47.

KALMAR, Deborah. Qué es la expresión Corporal: a partir de la corriente de trabajo creada por Patricia Stokoe. Buenos Aires: Lumen, 2005.

LAPLANTINE, François. La description ethnographique. Paris: Armand Colin, 2015.

LÓPEZ FERNÁNDEZ, Iván. Evolución histórica de la formación inicial del profesorado de educación física en España. Revista Fuentes, n. 4, 2003. Disponível em: https://revistascientificas.us.es/index. php/fuentes/article/download/2435/2304. Acceso en: Acceso en: 29 sept. 2015.

MAISONNEUVE, Jean; BRUCHON, Marilou. Modèles du corps et psychologie esthétique. Paris: PUF, 1981.

MANRIQUE, Juan Carlos. La Educación Física Femenina y el ideal de mujer en la etapa franquista. Revista Internacional de Medicina y Ciencias de la Actividad Física y el Deporte, v. 3, n. 10, p. 83-100, 2003. Disponível em: http://cdeporte.rediris.es/revista/revista10/artmujer.pdf. Acceso en: Acceso en: 29 sept. 2015.

MARCHESI, Álvaro. Presente y futuro de la reforma educativa en España. La Revista Iberoamericana de Educación, 2001. Disponível em: https://rieoei.org/historico/documentos/ rie27a03.htm. Acceso en: Acceso en: 29 sept. 2015.

MARTíN, Francisco Jesús. Origen y evolución de los contenidos en Educación Física y su relación con los estilos de enseñanza. Revista Digital Innovación y Experiencias Educativas, n. 18, p. 1-15, 2009. Disponível em: https://archivos.csif.es/archivos/andalucia/ensenanza/revistas/csicsif/revista/pdf/ Numero 18/FRANCISCO JESUS MARTIN RECIO02.pdf. Acceso en: Acceso en: 29 sept. 2015.

MATEU, M. Estudio de la lógica interna de los espectáculos artísticos profesionales: Cirque du Soleil ( 1986-2005 ). Universidad de Barcelona, 2010. Disponível em: http://www.tdx.cat/ handle/10803/132584. Acceso en: Acceso en: 29 sept. 2015. 
MONTÁVEZ, Mar. La expresión corporal en la realidad educativa: Descripción y análisis de su enseñanza como punto de referencia para la mejora de la calidad docente en los centros públicos de educación primaria de la ciudad de Córdoba. 2012. Tesis (doctoral) - Universidad de Córdoba, Córdoba, 2012.

ORTIZ, Ma Mar. Expresión Corporal Una propuesta didáctica para el profesorado de Educación Física. Granada: Grupo Editorial Universitario, 2002.

REBOLLO, José Antonio; CASTILLO, Estefanía. La Expresión Corporal como materia curricular del área de Educación Física para primaria. EF Deportes, v. 15, n. 146, p. 1-9, 2010. Disponível em: http://www.efdeportes.com/efd146/la-expresion-corporal-como-materia-de-educacion-fisica.htm. Acceso en: 21 oct. 2015.

RÍOS, Mercedes. Manual de Educación Física adaptada al alumno con discapacidad. Barcelona: Inde, 2006.

RIVERO, Antonio. Los valores del regeneracionismo en la educación física española (1898-1936). Revista Española de Educación Física y Deportes, n. 11, p. 13-25, 2009. Disponível em: http:// www.reefd.es/index.php/reefd/article/download/318/308. Acceso en: 15 nov. 2015.

ROMERO, Ma Rosario. Expresión corporal en Educación Física. Zaragoza: Prensas de la Universidad de Zaragoza, 2015.

RUANO, Kiki. La influencia de la expresión corporal sobre las emociones: un estudio experimental. 2004. Tesis (doctoral) - Universidad Politécnica de Madrid, Madrid, 2004.

SÁNCHEZ, Galo. La Escuela de Verano "Expresión, Creatividad y Movimiento" de Zamora. 10 años de proyectos compartidos. In: SÁNCHEZ, G. ; J. COTERÓN; PADILLA, C.;. RUANO, K. (Coords). Expresión Corporal, Investigación y Acción Pedagógica. Salamanca: Amarú Ediciones, 2008.

SÁNCHEZ, Galo. La Expresión Corporal-Danza en Patricia Stokoe. In: RUANO, K.; SÁNCHEZ, G. (Coords.) Expresión Corporal y Educación. Sevilla: Wanceulen Editorial Deportiva, 2009. p. 151174.

SÁNCHEZ, Galo; COTERÓN, Javier. Ana funda escuela. Revista de Educación y Biblioteca, n. 160, p. 92-96, 2007.

STOKOE, Patricia. Expresión Corporal-Danza: ese lenguaje silenciado. Revista Topía, n. 9, p. 1, nov.1993.

VÁZQUEZ, Benilde. La educación física en la educación básica. Madrid: Gymnos, 1989.

VIDAL BARBIER, Miguel; VIDAL ALMIÑANA, Miguel. Tendencias del pasado, presente y futuro de la Educación Física en España. Revista Española de Educación Física y Deportes, n. 9, p. 103-121, 2008. Disponível em: http://www.reefd.es/index.php/reefd/article/download/340/330.Acceso en: 29 oct. 2016.

Agradecemos el apoyo y asesoramiento prestados para la elaboración de este artículo a los profesores Galo Sánchez, María Rosario Romero, Mikel Chivite y Mercè Mateu, así como al profesorado experto de Expresión Corporal que ha participado de este estudio. 